\title{
Crescent MOONS: an update on the ongoing construction of the new VLT's multi-object spectrograph
}

\author{
Cirasuolo, Michele, Gonzalez, Oscar, Rees, Phil, Bryson, \\ Ian, Fairley, Alasdair, et al.
}

Michele Cirasuolo, Oscar Gonzalez, Phil Rees, Ian Bryson, Alasdair Fairley, William Taylor, Jose Afonso, Simon Lilly, Chris Evans, Hector Flores, Roberto Maiolino, Ernesto Oliva, Stephane Paltani, Leonardo Vanzi, Manuel Abreu, Jean-Philippe Amans, David Atkinson, Steven Beard, Clementine Béchet, Andrea Belfiore, Martin Black, David Boettger, Saskia Brierley, David Buscher, Alexandre Cabral, Marcella Carollo, William Cochrane, João Coelho, Miriam Colling, Ralf Conzelmann, Francesco Dalessio, Louise Dauvin, George Davidson, Giles Fasola, Debora Ferruzzi, Martin Fisher, Mauricio Flores, Bianca Garilli, Adriana Gargiulo, Julien Gaudemard, Isabelle Guinouard, Pablo Gutierrez, Régis Haigron, Chris Haniff, Derek Ives, Olaf Iwert, David King, Suzanne Kovacz, Philippe Laporte, David Lee, Gianluca Li Causi, Yerko Luco, Alastair Macleod, Charles Maire, Basile-Thierry Melse, António Oliveira, Livia Origlia, lan Parry, Fernando Pedichini, Roberto Piazzesi, Florent Reix, Myriam Rodrigues, Felipe Rojas, Frédéric Royer, Pedro Santos, Robin Schnell, Tzu-Chiang Shen, Michael Sordet, Jonathan Strachan, Xaiowei Sun, Graham Tait, Andrea Tozzi, Simon Tulloch, Lauren Von Dran, Chris Waring, Stephen Watson, Brian Woodward, Yanbin Yang, "Crescent MOONS: an update on the ongoing construction of the new VLT's multi-object spectrograph," Proc. SPIE 11447, Ground-based and Airborne Instrumentation for Astronomy VIII, 1144717 (13 December 2020); doi: 10.1117/12.2561229 


\title{
Crescent MOONS: an update on the ongoing construction of the new VLT's multi-object spectrograph
}

Michele Cirasuolo $^{\mathrm{a}}$, Oscar Gonzalez, Phil Rees ${ }^{\mathrm{b}}$, Ian Bryson ${ }^{\mathrm{b}}$, Alasdair Fairley ${ }^{\mathrm{b}}$, William Taylor ${ }^{\mathrm{b}}$, Jose Afonso $^{\mathrm{c}, \mathrm{d}}$, Simon Lilly ${ }^{\mathrm{e}}$, Chris Evans ${ }^{\mathrm{b}}$, Hector Flores ${ }^{\mathrm{f}}$, Roberto Maiolino ${ }^{\mathrm{g}}$, Ernesto Oliva ${ }^{\mathrm{h}}$, Stephane Paltani, Leonardo Vanzi ${ }^{j}$, Manuel Abreu ${ }^{c, d}$, Jean-Philippe Amans ${ }^{\mathrm{f}}$, David Atkinson ${ }^{\mathrm{a}}$, Steven Beard ${ }^{\mathrm{a}}$, Clementine Béchet ${ }^{\mathrm{j}}$, Andrea Belfiore $^{\mathrm{m}}$, Martin Black ${ }^{\mathrm{a}}$, David Boettger ${ }^{\mathrm{j}}$, Saskia Brierley ${ }^{\mathrm{a}}$, David Buscher ${ }^{\mathrm{g}}$, Alexandre Cabral ${ }^{\mathrm{c}, \mathrm{d}}$, Marcella Carollo ${ }^{\mathrm{e}}$, William Cochrane ${ }^{\mathrm{a}}$, João Coelho ${ }^{\mathrm{c}, \mathrm{d}}$, Miriam Colling ${ }^{\mathrm{k}}$, Ralf Conzelmann ${ }^{\mathrm{b}}$, Francesco Dalessio $^{1}$, Louise Dauvin ${ }^{\mathrm{j}}$, George Davidson ${ }^{\mathrm{a}}$, Giles Fasola ${ }^{\mathrm{f}}$, Debora Ferruzzi ${ }^{\mathrm{h}}$, Martin Fisher ${ }^{\mathrm{g}}$, Mauricio Flores ${ }^{\mathrm{j}}$, Bianca Garilli $^{\mathrm{m}}$, Adriana Gargiulo ${ }^{\mathrm{m}}$, Julien Gaudemard ${ }^{\mathrm{f}}$, Isabelle Guinouard ${ }^{\mathrm{f}}$, Pablo Gutierrez ${ }^{\mathrm{b}}$, Régis Haigron ${ }^{\mathrm{f}}$, Chris Haniff ${ }^{\mathrm{g}}$, , Derek Ives ${ }^{\mathrm{b}}$, Olaf Iwert ${ }^{\mathrm{b}}$, David King ${ }^{\mathrm{p}}$, Suzanne Kovacz ${ }^{\mathrm{b}}$, Philippe Laporte ${ }^{\mathrm{f}}$, David Lee ${ }^{\mathrm{a}}$, Gianluca Li Causi ${ }^{1}$, Yerko Luco ${ }^{\mathrm{j}}$, Alastair Macleod ${ }^{\mathrm{a}}$, Charles Maire ${ }^{\mathrm{i}}$, Basile-Thierry Melse ${ }^{\mathrm{f}}$, António Oliveira $^{\mathrm{c}, \mathrm{d}}$, Livia Origlia ${ }^{\mathrm{o}}$, Ian Parry ${ }^{\mathrm{p}}$, Fernando Pedichini ${ }^{1}$, Roberto Piazzesi ${ }^{1}$, Florent Reix ${ }^{\mathrm{f}}$, Myriam Rodrigues $^{\mathrm{f}}$, Felipe Rojas ${ }^{\mathrm{j}}$, , Frédéric Royer ${ }^{\mathrm{f}}$, Pedro Santos ${ }^{\mathrm{c}, \mathrm{d}}$, Robin Schnell, Tzu-Chiang Shen ${ }^{\mathrm{q}}$, Michael Sordet ${ }^{\mathrm{i}}$, Jonathan Strachan ${ }^{\mathrm{a}}$, Xaiowei Sun ${ }^{\mathrm{g}}$, Graham Tait ${ }^{\mathrm{a}}$, , Andrea Tozzi ${ }^{\mathrm{h}}$, Simon Tulloch ${ }^{\mathrm{b}}$, Lauren Von Dran ${ }^{\mathrm{k}}$, Chris Waring ${ }^{\mathrm{a}}$, Stephen Watson ${ }^{\mathrm{a}}$, Brian Woodward ${ }^{\mathrm{a}}$, Yanbin Yang ${ }^{\mathrm{f}}$

${ }^{\text {a }}$ ESO, Karl-Schwarzschild-Str. 2, 85748, Garching bei München, Germany; ${ }^{\mathrm{b}}$ STFC, UK Astronomy Technology Centre, Royal Observatory Edinburgh, Edinburgh, EH9 3HJ, UK; ${ }^{c}$ Instituto de Astrofísica e Ciências do Espaço, Universidade de Lisboa, Campus do Lumiar, Estrada do Paço do Lumiar 22, Edif. D, PT1649-038 Lisboa,Portugal; ${ }^{\text {d}}$ Laboratório de Óptica, Lasers e Sistemas, Departamento de Física, Faculdade de Ciências, Universidade de Lisboa, Campo Grande 1749-016 Lisboa, Portugal; ${ }^{\text {E }}$ ETH Zurich, Department of Physics, Wolfgang-Pauli-Strasse 27, 8093 Zurich, Switzerland; ${ }^{\mathrm{f}}$ GEPI, CNRS, Observatoire de Paris, PSL University, France; ${ }^{\mathrm{g}}$ Department of Physics, Cavendish Laboratory, JJ Thomson Avenue, Cambridge, CB3

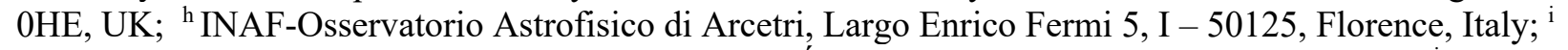
Department of Astronomy, University of Geneva, ch. d'Écogia 16, CH-1290 Versoix, Switzerland; ${ }^{j}$ Pontificia

Universidad Católica de Chile, Av. Vicuña Mackenna 4860, 7820436 Macul, Santiago, Chile; ${ }^{k}$ STFC,

Rutherford Appleton Laboratory, Harwell Campus, Oxfordshire, OX11 0QX, UK; ${ }^{1}$ INAF-Osservatorio

Astronomico, Viale del Parco Mellini 84, Roma, Italy; ${ }^{\mathrm{m}}$ INAF - IASF Milano, Via E. Bassini 15, I-20133

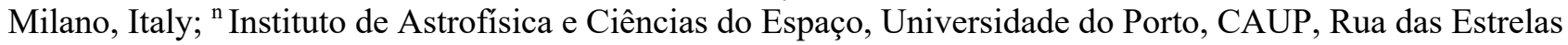
4150-762 Porto, Portugal; ${ }^{\circ}$ Instituto di astrofisica spaziale e fisica cosmica di Bologna, Area della Ricerca via Piero Gobette, 101 - 400129, Bologna, Italy; ${ }^{\mathrm{p}}$ Institute of Astronomy, University of Cambridge, Madingley Road, Cambridge, CB3 0HA, UK; ${ }^{9}$ BlueShadows Ltda., Barros Errazurriz, 1954, OF 706, Sanatigo, Chile

\begin{abstract}
The Multi Object Optical and Near-infrared Spectrograph (MOONS) instrument is the next generation multi-object spectrograph for the VLT. This powerful instrument will combine for the first time: the large collecting power of the VLT with a high multipexing capability offered by 1000 optical fibres moved with individual robotic positioners and a novel, very fast spectrograph able to provide both low- and high-resolution spectroscopy simultaneously across the wavelength range $0.64 \mu \mathrm{m}-1.8 \mu \mathrm{m}$. Such a facility will provide the astronomical community with a powerful, world-leading instrument able to serve a wide range of Galactic, Extragalactic and Cosmological studies. The final assembly, integration and verification phase of the instrument is now about to start performance testing.
\end{abstract}

Keywords: Multi-object Spectrograph, Optical and near-IR spectrograph, medium and high-resolution spectrograph, Galactic surveys, Extragalactic surveys

*mciras@eso.org wwww.vltmoons.org

Ground-based and Airborne Instrumentation for Astronomy VIII, edited by Christopher J. Evans,

Julia J. Bryant, Kentaro Motohara, Proc. of SPIE Vol. 11447, 1144717 · (c) 2020

SPIE · CCC code: $0277-786 \mathrm{X} / 20 / \$ 21 \cdot$ doi: $10.1117 / 12.2561229$ 


\section{INTRODUCTION}

Over the last two decades several key observational efforts have dramatically changed our knowledge of the Universe. Yet, some of the most fundamental problems in science remain to be solved: Understanding the nature of the dark components which dominate the global expansion and Large-Scale Structure of the Universe and the physical processes that affect baryons and shape the formation and evolution of stars and galaxies. Answering these questions demands an accurate reconstruction of the assembly history of stars and galaxies over virtually all cosmic times in order to decode the building blocks of the Universe.

The Milky Way offers a one-of-a-kind opportunity to reconstruct the assembly history of a prototypical spiral galaxy by looking at the individual chemical abundances, ages, and orbital movements of its stellar populations down to its innermost components. On the other hand, tracing the evolution of galaxy properties beyond our own galaxy (star-formation, metallicity, mass-assembly, etc.), over the whole cosmic epoch, is key to understand the effects of age and environment. In fact, pushing these studies to the highest redshifts - when the Universe was just a few hundred million years old provides the opportunity to investigate the physics of the early Universe and cosmic re-ionization. These ambitious science goals require an accurate determination of stellar and galactic physical properties for as many stars and galaxies as possible.

To this end, the MOONS instrument has been developed focusing on 3 essential requirements: sensitivity, multiplex and wavelength coverage. The high sensitivity is being achieved by optimising all the components of the MOONS instrument for high transmission that is coupled with the large aperture and efficiency of the VLT. The multiplex of 1000 is a factor 20 more than current spectrographs operating in the near-infrared, thus pushing the limit on budget, technical capability to manufacture the very large optics, as well as the mass and volume available at the Nasmyth platform for the large MOONS cryostat.

Finally, the broad wavelength coverage of MOONS of $0.645 \mu \mathrm{m}-1.8 \mu \mathrm{m}$, extending in the near-IR is critical to observe heavily dust-obscured regions of our Milky Way as well to open the window onto the high-redshift Universe. To meet the aspirations of both the Galactic and Extragalactic scientific communities, MOONS offers both low- and high-resolution spectroscopy. In the low-resolution mode $(\mathrm{R} \sim 4,000-6,000)$, the entire $0.645-1.8 \mu \mathrm{m}$ range is observed simultaneously, across the RI, YJ and $\mathrm{H}$ atmospheric windows. In the high-resolution mode three carefully selected windows are simultaneously covered: the $\mathrm{YJ}$ channel that remains at $\mathrm{R} \sim 4000$, an $\mathrm{RI}$ channel with $\mathrm{R}>9,000$ around the CaT region to measure stellar radial velocities, and another with $\mathrm{R} \sim 19,000$ in the H-bands for detailed measurements of chemical abundances. Details on the instrument specifications can be found at www.vltmoons.org.

\section{INSTRUMENT OVERVIEW}

\subsection{Technical summary}

MOONS is a fibre-fed, optical to near-IR, multi-object spectrograph designed to use the full 25 arcmin diameter field-ofview (FoV) of one of the Unit Telescopes (UT) of the VLT. The instrument is made of 3 major sub-systems shown in Figure 1: the part that is mechanically attached to the telescope and couples the light into the optical fibres called the rotating front end (RFE), the two triple-arm spectrographs wherein the light from the fibres is dispersed and recorded, and the instrument control.

The fibres for science observations are deployed on the focal plane created by the Field Corrector using 1001 miniature Fibre Positioning Units (FPU), allowing us to configure an entire observation in less than 2 minutes. Each fibre is connected to its own pick-off unit, which has a footprint of $25 \mathrm{~mm}$ fixed on the focal plane and is equipped with two rotating arms. The combination of the two rotations (like the combined motion of elbow and shoulder) allows the fibre to patrol an area with a diameter of $50 \mathrm{~mm}(\sim 1.5 \mathrm{arcmin}$ on sky). The FPUs can achieve high positioning accuracy using state-of-the art stepper-motors, but in order to monitor this and make any calibration adjustments there is also an external metrology system capable of precisely measuring the position of each fibre. On the focal plane, spread out amongst the FPUs, there are 20 acquisition cameras used to acquire the science field and do a fine alignment of the instrument on sky. The rotating front end also hosts a novel concept of calibration unit, which uses a projector to illuminate a screen coated in a Lambertian diffuser to guarantee high quality wavelength calibration and flat fielding for all fibres. Indeed, to ensure excellent sky- 
subtraction it is critical that the relative transmission of all the fibres is known to very accurately, better than $1 \%$, and this highly homogeneous illumination is achieved via the calibration unit.

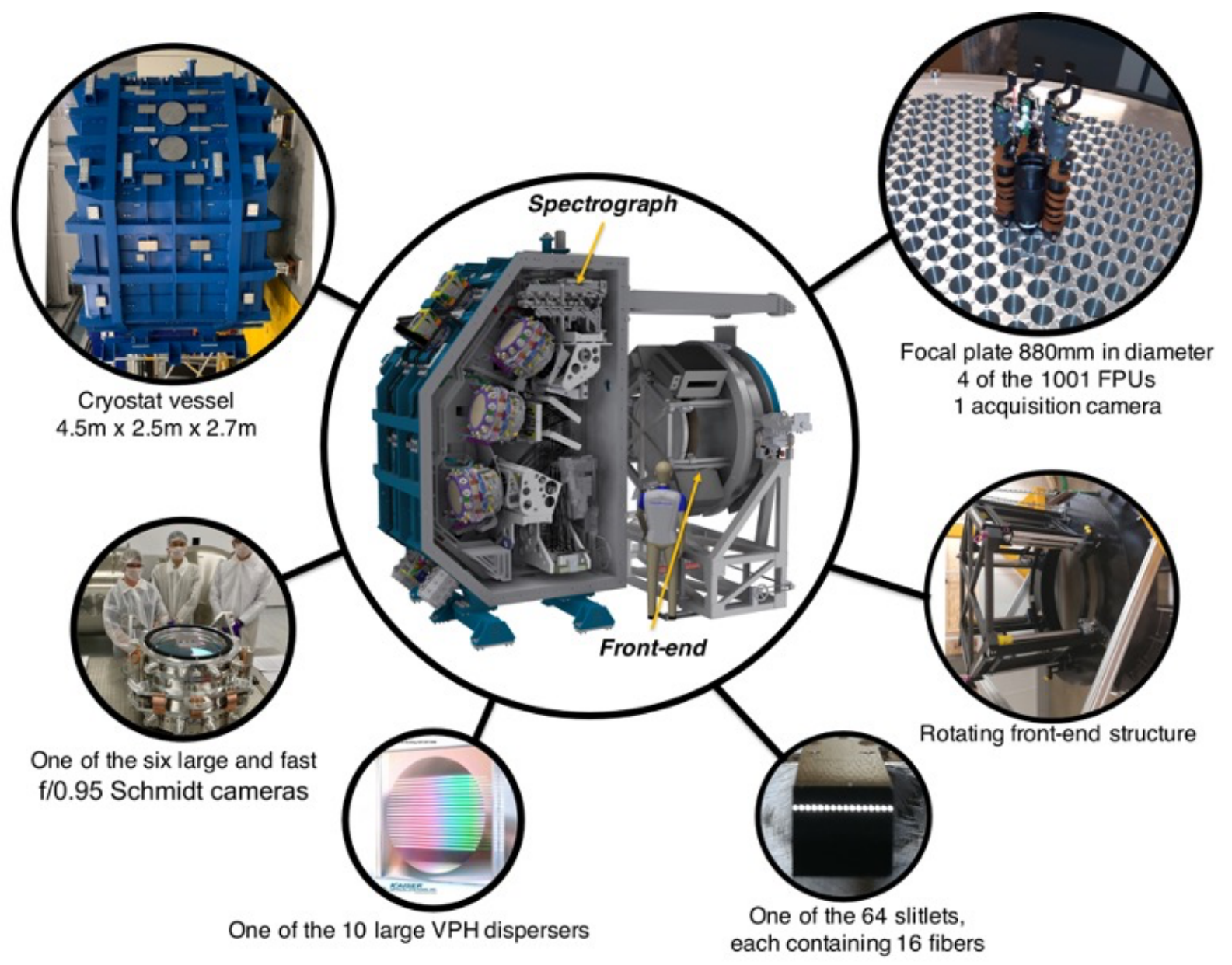

Figure 1 The central figure shows the CAD model of the MOONS instrument, highlighted the rotating front-end and the spectrograph. The various images around show the real hardware being integrated. From top right clock-wise: The focal plate with 4 mounted fibre positioning units (FPUs) and one acuisition camera; The structure of the rotating front-end that will host the plate with the FPUs, the calibration unit and the metrology system; one of the slitlet with 16 mounted fibres; one of the 10 high efficiency VPH-disperser; one of the 6 Schmidt cameras, the cryostat vessel.

Once the light from stars and galaxies is collected at the front end, it is then fed through the fibres to two identical, triple arm spectrographs enclosed in a single cryostat vessel that keeps the optical elements inside at a temperature of $140 \mathrm{~K}$ in order to reduce the background in the near-IR. In each of the two spectrographs the light from 512 fibres - arranged in 32 slitlets each containing 16 fibres (see Figure 1) - is split by dichroic filters into three wavelength ranges or channels (RI, YJ and H). Each of the two MOONS spectrographs has 5 highly efficient Volume Phase Holographic (VPH) dispersers, 3 low-res and 2 high-res. The two triple-arm spectrographs are mounted back-to-back on the optical bench, which makes it is possible to switch between the high- and low-resolution modes in the RI-band (and similarly for the H-band) using a single common linear mechanism that passes straight through the optical bench. In each channel, the light dispersed by the VPHs is refocused by using Schmidt-like cameras with an f-number of $\mathrm{f} / 0.95$ which are the fastest (to our knowledge) large cameras ever built-in astronomy. Each camera is also very compact and made of just two lenses (glued one inside the other) and one mirror to bring the image into focus on a detector (see Figure 1), and therefore easy to align. Finally, the light (that travelled in some cases for billions of years) will be recorded on state-of-the-art detectors. The two infra-red 
channels (YJ and $\mathrm{H}$ ) will exploit the new Hawaii 4RGs $15 \mu \mathrm{m}$ pixels detectors and the optical channel (RI) with fullydepleted LBNL red-sensitive CCDs.

\subsection{Observing Strategies}

Accurate subtraction of the sky background is critical when observing faint sources, particularly in the near-IR, where strong $\mathrm{OH}$ sky-lines dominate the background. To achieve this goal, we have implemented multiple methods. First of all, the spectral resolving power of $\mathrm{R}>4,000$ for the medium-resolution mode $(\mathrm{R}>6500$ in H-band) ensures that at least 60$70 \%$ of the observed regions in the YJ- or H-bands are completely free from $\mathrm{OH}$ airglow. Sky subtraction with fibres is challenging since they might change their efficiency (even slightly) when they move. For this reason, particular attention has been devoted during the manufacturing of the fibres and their routing within the instrument in order to minimize the variation of focal ratio degradation (FRD), which has been measured to be $<<1 \%$. In order to remove any residual, it is also possible to obtain a fast attached flat after the fibres have been reconfigured and are in their science position. Still, in order to further optimize the sky subtraction, the fibre positioners have been designed to have overlapping patrol fields and capable of be placed at 10 arcsec distance, to be able to sample simultaneously the sky very close to the science target.

This is also driving the 3 possible observing strategies envisaged for MOONS (see Figure 2). During the commissioning these strategies will be tested, and the performance of sky-subtraction evaluated, in order to provide guidelines for the users.

Stare: In this observing strategy the vast majority of fibres will be on targets, with dedicated sky fibres distributed across the focal plane. The number of sky fibres can be determined by the user.

Stare+Nod: The majority, if not all fibres will be on targets, and the telescope is then nodded to a nearby sky position. This has the advantage that the sky flux will pass through the same fibre as the target, thus removing many instrumental effects. The quality of the sky-subtraction will depend on the frequency of the sky nods.

XSwitch: This provides a pseudo-slit observation, with the most accurate sky-subtraction. Every science fibre will have an adjacent sky fibre at the same fixed distance $\left(10^{\prime \prime}<\mathrm{d}<30^{\prime \prime}\right)$ and same direction. The telescope is then nodded by the same distance and direction, so that object and sky fibres are reversed. This observing pattern strategy allows both temporal and spatial sky variation to be removed, as well as accounting for instrumental effects.
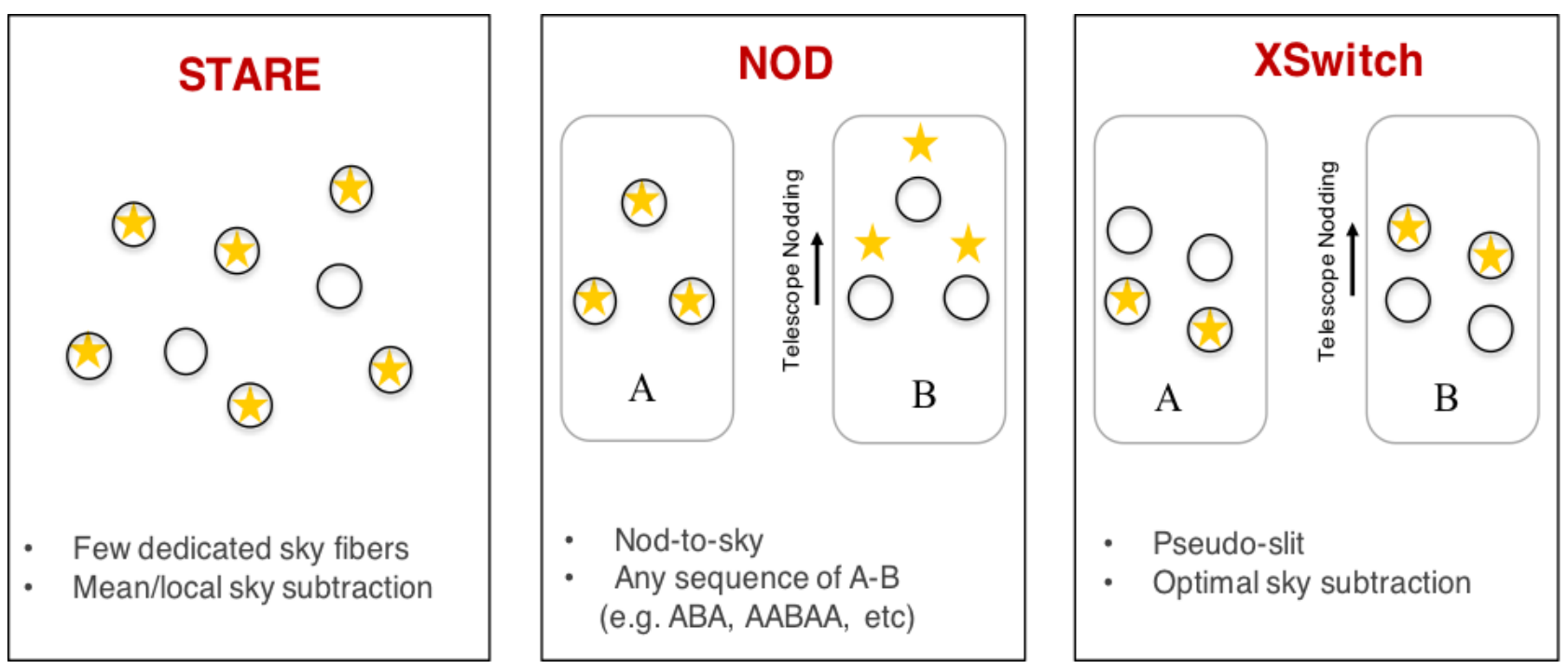

Figure 2. The three possible observing strategies envisaged for MOONS in terms of sky-subtraction. 


\section{INSTRUMENT STATUS UPDATE}

\subsection{The rotating front-end structure and field corrector}

The MOONS rotating front-end (RFE) is the large assembly that will be mounted on the VLT Nasmyth rotator and on the other end connected to the two static Spectrographs via fibre assemblies. The system includes a retractor mechanism that is in charge of retracting the entire fibre positioning support structure for metrology purposes and to provide the required distance from the calibration screen during instrument calibrations. The calibration screen is located close to the field corrector and is controlled by two sliding doors that can be closed to form an $880 \mathrm{~mm}$ diameter screen.

All the components of the MOONS RFE Structure, after manufacturing by CUNHOL (Portugal), are now at the IA (Lisbon) MOONS integration room, described in [1]. The entire main structure, including its retraction and screen mechanisms, have been fully integrated, see Figure 3. Both the RFE Structure and the Retraction mechanism were tested qualitatively with results fully meeting the requirements. Load tests were successfully performed with $330 \mathrm{~kg}$ (Focal Plate weight) in order to fully characterize the behavior of the plate positioning both in the Science and Metrology positions.

The first component that optically interfaces MOONS with the VLT, is the Field Corrector (see [1]). The field corrector ensures that the exit pupil of the VLT is almost concentric to the focal plane's curvature. It also changes the radius of curvature of the focal plane from $2090 \mathrm{~mm}$ to $4210 \mathrm{~mm}$; thus, making the focal plane closer to a flat surface, which significantly simplifies the assembly of the units on the focal plate - both the back and the front surfaces.

The field corrector is being manufactured at Officina Stellare (Italy). The lenses L1 and L2 are ready and their polishing has been completed. Currently the lenses are ready for the Anti-Reflection coating, followed by their integration in the respective barrels.
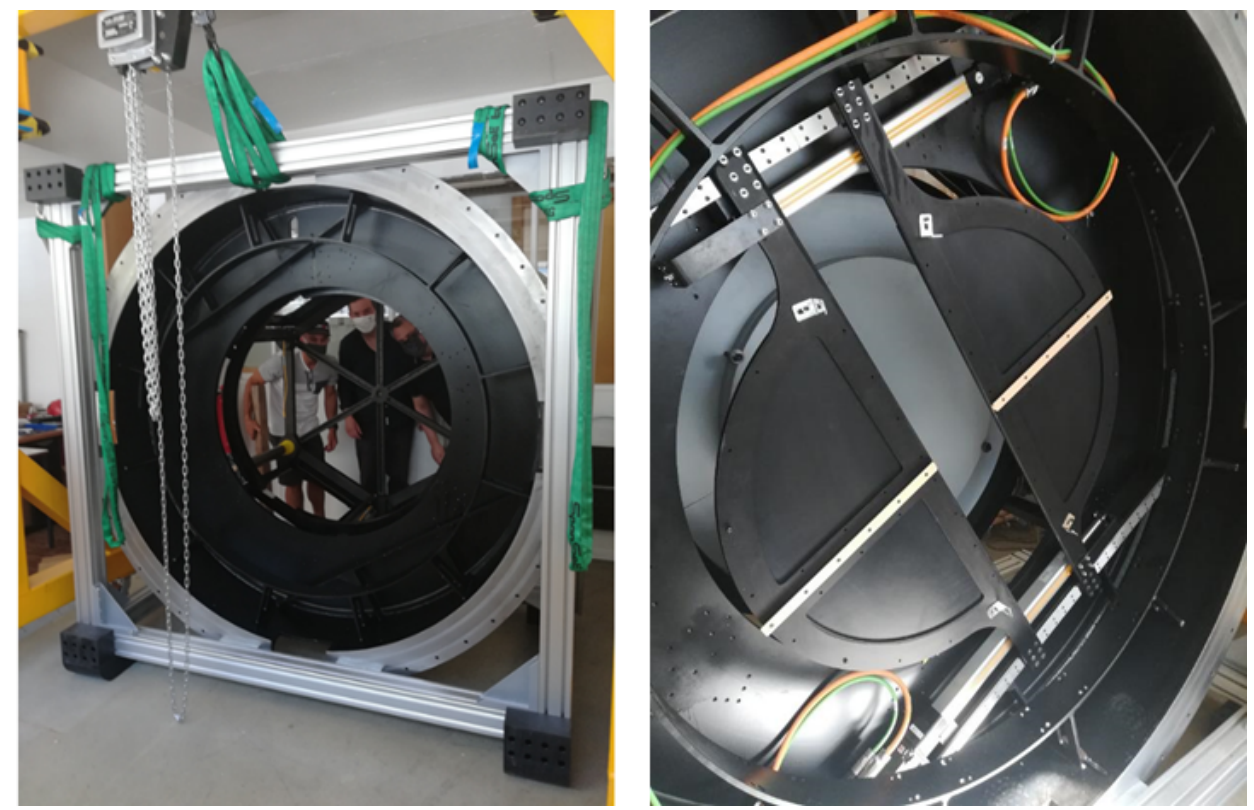

Figure 3. Rotating front end structure during integration and tests of the retraction and screen mechanisms

\subsection{Focal plate: Fibre Position Units and Acquisition Cameras}

The $\sim 1 \mathrm{~m}$ diameter MOONS focal plate holds both the fibre positioning system and the Acquisition Cameras. A set of fiducial markers are also installed in the plate as reference for metrology measurements. The system has a total of $20 \mathrm{ACs}$, 48 fiducials, and 1001 FPUs distributed in the field.

Each FPU has two stepper motors, which drive an alpha and beta arm that act like an elbow and shoulder joint to give complete coverage over a small region of the focal plane. The FPUs are jointly developed by the MPS group in Switzerland and the UKATC. Working closely together through the design and testing stages, the high-performance requirements of 
these units have now been met. Their key design features and a detailed description of the calibration and testing procedures are described in [2].

The main science driver for the tight requirement tolerances for the FPUs, particularly on tilt alignment, comes from the need for excellent sky-subtraction of MOONS on very faint sources. The best sky subtraction in MOONS can be achieved by bringing FPUs close together in pairs to within 10 arcsecond separation on sky (in X-switch mode) offsetting between a fibre on target and one on sky to map the strong spatial and temporal variations of the near-IR sky. To achieve this, each FPU must be optimally aligned with the telescope pupil, since failure to achieve this would make impossible to calibrate the fibre-to-fibre variation in transmission. The FPUs in production have an optical alignment of $0.12^{\circ}$, which is better than the $20^{\circ}$ requirement.

Similarly, since MOONS does not have an Atmospheric Dispersion Corrector (ADC), the fibre aperture needs to be optimally aligned with the science source position on sky to avoid high losses during observations at high airmass at the edges of the wavelength range covered by MOONS. The measured positional repeatability of the FPUs (95th percentile confidence) in open loop, counting steps from the datum switch, is $11.4 \mu \mathrm{m}$. This translates into an excellent $0.03 \mathrm{arcsec}$ precision on sky positioning (from a $0.05 \mathrm{arcsec}$ requirement).

On the other hand, the overall instrument focal plane alignment with the field is handled by the Acquisition Camera system. Each of the $20 \mathrm{AC}$ has a 37"x37" field of view (30"x30" unvignetted) and can reach a R=21 mag star in less than 30s exposure. The combination of the AC distribution in the field and their sensitivity ensures that at least 2-3 stars are always available when selecting the optimal rotator angle. The acquisition stars are used to compute the pointing and rotator offsets needed to optimally align the fibres to their expected sky target positions. This is handled by a dedicated local Linux microcomputer (based on the Beagle Bone Black standard) that manages the exposures and processes the images to measure the relevant quantities on the acquisition stars (centroid, magnitude and FWHM) to be returned upon request to the MOONS Instrument Control Software. The Instrument Control Software then handles the computation of offsets either automatically or via interaction with the Instrument Operator.

All acquisition cameras are now completed with the remaining activities focusing on their integration into the ESO TCDS environment and the overall Instrument Control Software, as well as the refining of all user interfaces.

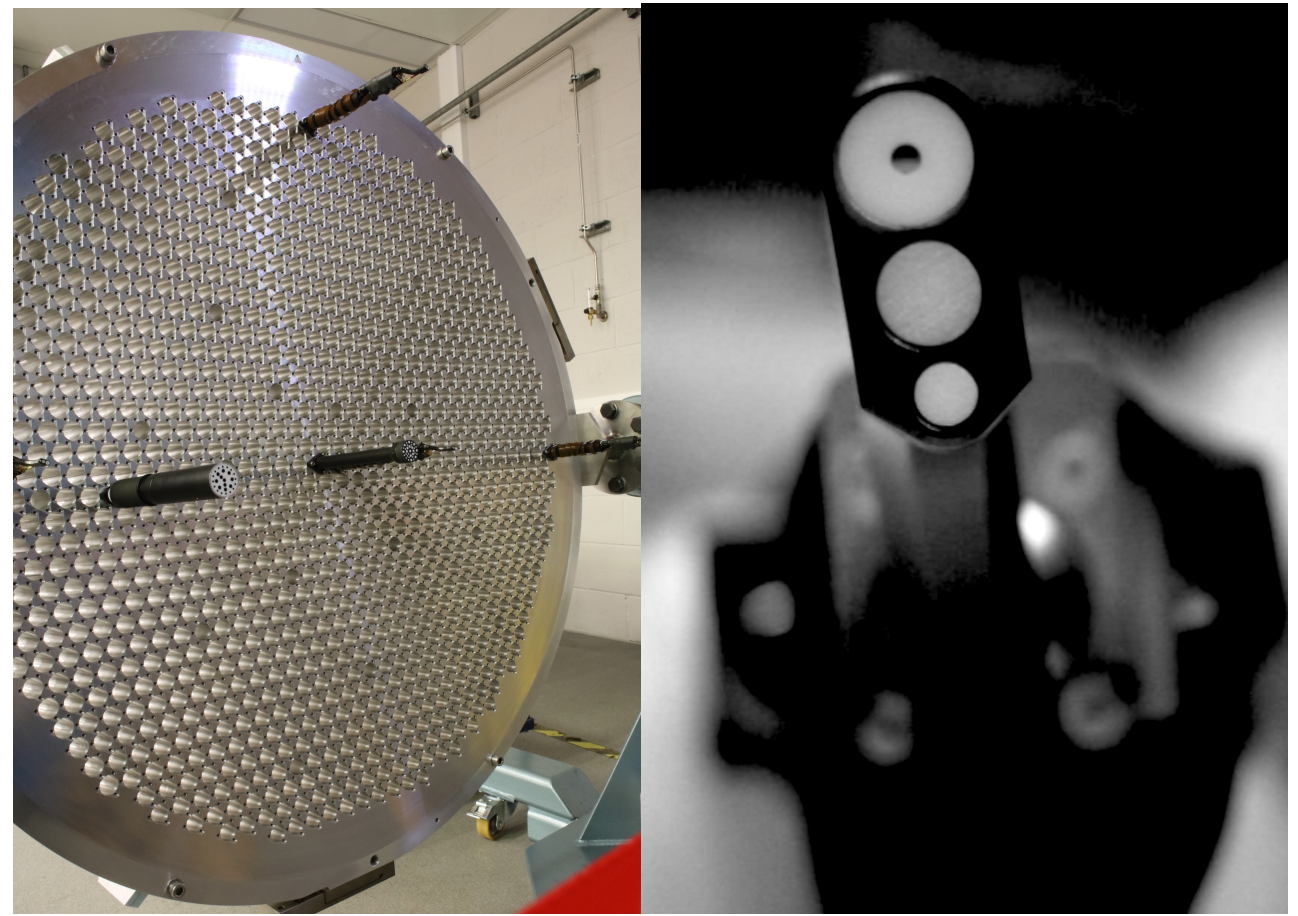

Figure 4. Left the MOONS focal plate with mounted 3 FPUs, an acquisition camera and a fiducial. Right: top view of one FPU, showing the MACOR field stop (top) and the two dots used by the metrology system. 


\subsection{Metrology System}

The MOONS metrology sub-system is designed to accurately determine the physical location of the fibre positioning units in the plate reference system at any point during operations. The most important function of the metrology system is to provide a means of recovery following software or hardware failure which leaves the fibre positioners in an unknown condition. The system will need to determine both the fibre position and angle of the beta-arms in order to safely unpick the field. In addition, the system provides the option of a quasiclosed loop control of the fibre positioners in case of aging related accuracy problems. Life testing of the FPUs suggests that this will not be needed, however a possible wear in the gears-box could reduce the repeatability of the FPUs. Therefore, this quasi-closed loop option is only likely to be needed after several years of use.

A new baseline for the metrology system has been defined, using an off-the-shelf photogrammetry system by Hexagon adapted to the MOONS RFE and corresponding software interfaces. The Canon 5DS camera is mounted on the RFE on a support that can rotate in a circle above the target plate to produce a set of images of the entire plate from different orientations. These will be analysed with the dedicated API photogrammetry analysis by Hexagon to recover the 3D physical locations of the reflective dots and the MACOR field stop installed in the beta-arms of the FPUs. The detected dots are then used to identify and recover the fibre locations and the arm angle which are then used by the Instrument Control software to recover the status of the FPUs. The accuracy in the position of the fibres obtained with this system is about $10-15 \mu \mathrm{m}$.

\subsection{Calibration Unit}

The MOONS calibration unit provides both flat fielding and wavelength calibration for all the fibres. When the focal plate is retracted into its calibration position and the screen is closed, each of the fibres will receive flux from an effective 30 $\mathrm{mm}$ diameter region of the screen's surface. This provides the instrument with flat-field illumination with which to measure the throughput of each fibre (to within 1\%), and illumination from a source of known wavelength (a ThAr lamp) with which to calibrate the wavelength scale of the spectrograph pixels. The flatness requirement for the flat-field illumination comes - same as the case of the FPUs - from the need for excellent sky subtraction (better than 1\%). To achieve this, the spatial variation of the flux across the calibration screen must also be equally flat over the $30 \mathrm{~mm}$ scale-lengths viewed by each fibre. To achieve this level of flatness MOONS is using a spatially uniform light source and dedicated software which uses a calibrated "truth" camera to correct for flat-field uniformity errors.

In addition to the regular daytime flat calibrations, attached flat fields to the science observations can be obtained once the FPUs are in their observing positions, providing a very accurate fibre-to-fibre relative transmission to account for possible FRD variations caused by the movement of the fibres. It is however reassuring that all the tests performed indicate that moving the fibres to different positions does not produce any measurable FRD effects.

The calibration unit has been fully assembled and it is now in the final stage of testing the control software and the flatness performance using the Lambertian screen, see Figure 5 and fully described in [3].

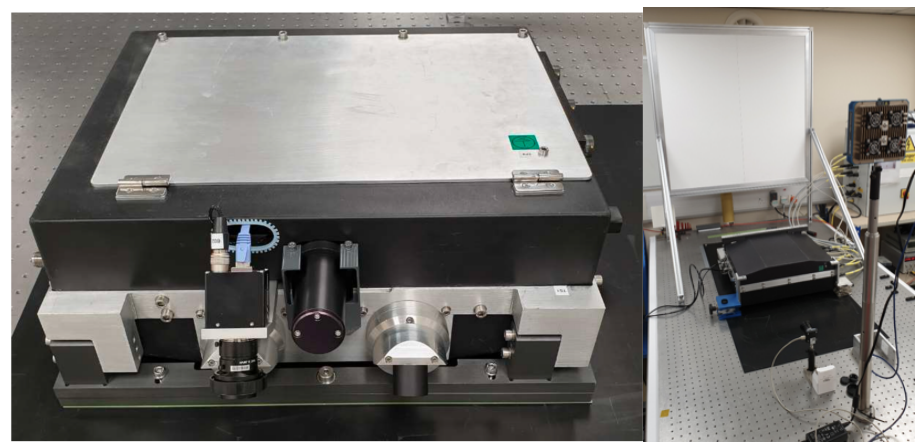

Figure 5. MOONS calibration unit. 


\subsection{Front-end fibre assemblies}

Fibres are used to pick-off each sub-field and to transport the light from the instrument focal plane to the two Spectrographs. The front-end fibre assembly houses the micro-lens, that couples the light into the corresponding fibre, glued to the fibre ferrule, both housed in the 1.2" field stop located at the end of the beta arm of the FPUs. The lens is slightly behind the focal plane with a field stop to ensure that the fibre is fed cleanly. A recent design change was implemented by making the field stop in white MACOR ceramic, thus making it available as a target for the metrology system and substantially reducing the error budget by directly measuring the fibre position.

The assembly of the front-end fibres is now well underway, more information provided in [4]. Testing of the first batch of assemblies show them to be well within the tilt specification of 6 arcmin. The mean value of 15 tested assemblies is 2.6 arcmin. Even without active gluing of the field stop, the error is within the specification.

\subsection{Cryostat}

The two triple-arm spectrographs are mounted on a central vertical optical bench, whose natural symmetry reduces stresses within the system. The cryostat has a central section that surrounds the optical bench and on either side of this are two large doors covering each of the two spectrographs. The cryostat operates at around $130 \mathrm{~K}$ and its first full cooldown to confirm thermal performance and associated control processes has now been successfully completed.

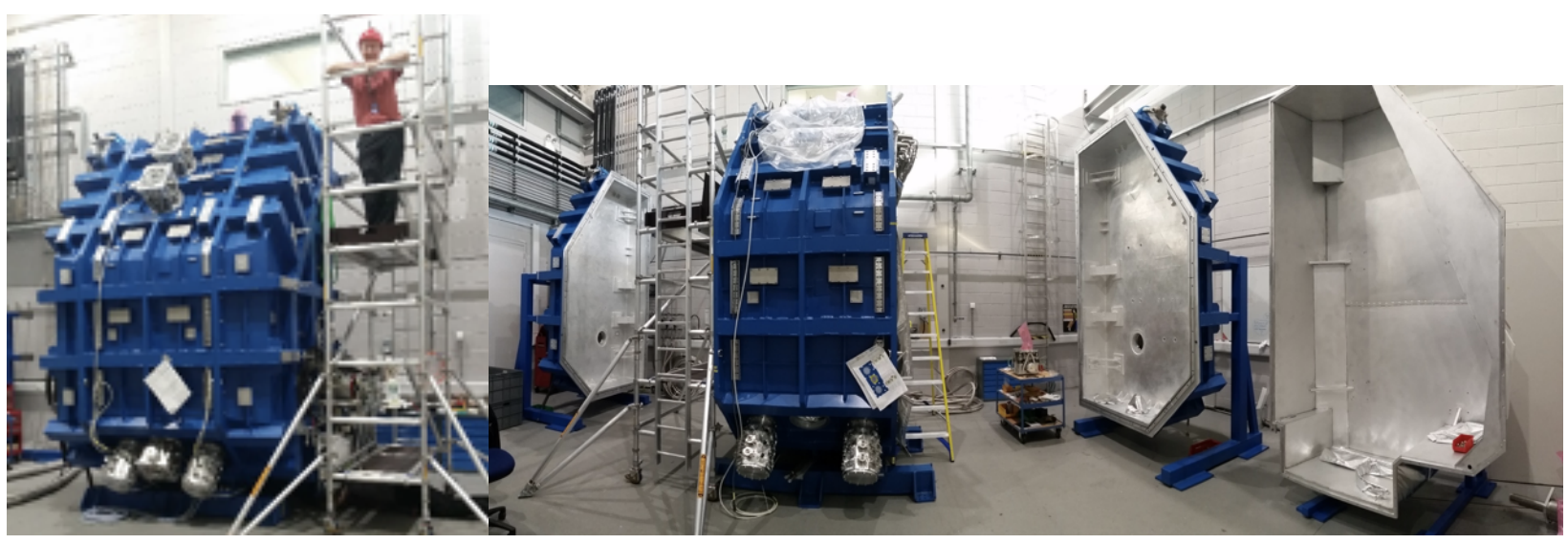

Figure 6. The MOONS cryostat ( $4.2 \times 2.5 \times 2.7$ metres), on the left fully assembled, while on the right showing the two sections opened.

\subsection{Slit and fibre assembly}

MOONS has two slits, one for each spectrograph, comprised of 32 slitlets. Each slitlet is manufactured from wire-eroded Invar and contains 16 fibres. More than $75 \%$ of the fibre bundles have been received and tested, showing an excellent transmission, better than requirement as detailed in [5]. The slitlets have a V-groove profile into which the fibres are laid before being secured with a flat substrate that is fastened on top of them. The first slit comprising 512 fibers fitted through the vacuum-feeds has been completed and the final alignment of the slitlets is being completed.

\subsection{Cameras}

Due to the large number of fibres and size of the available detectors MOONS requires fast, large optics: all the spectral channels consequently use similar f/0.95 Schmidt cameras, to our knowledge the fastest large cameras ever built in astronomy. All the optical elements, the mirrors and the L1/L2 lens assembly (glued together) have been completed and delivered by Winlight. The First camera has been fully assembled and successfully aligned in cold at operating temperature of $130 \mathrm{~K}$. Initially it was aligned warm and then the whole test assembly inserted 
into the cryostat for final cold alignment followed by thermal cycling successfully demonstrating the alignment stability.

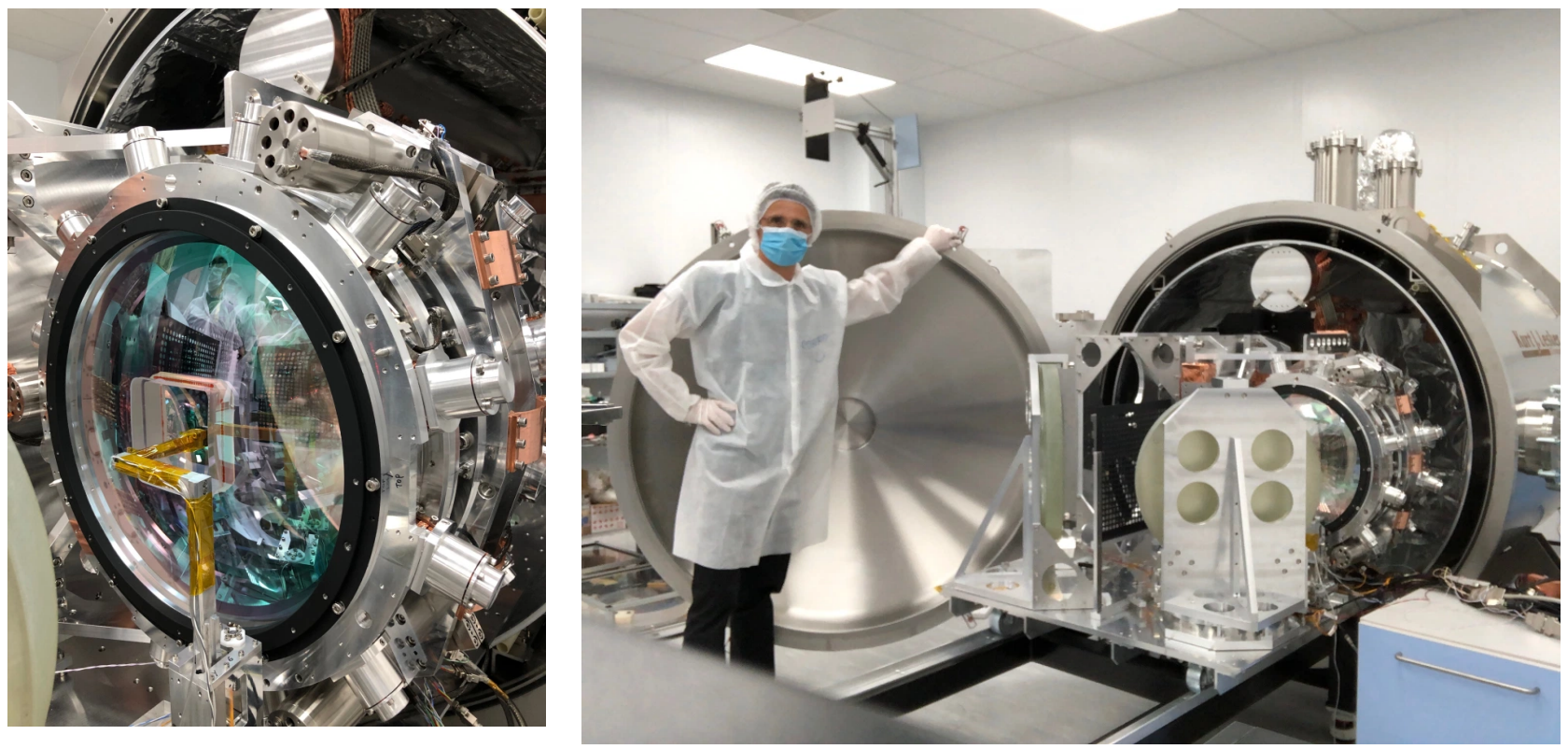

Figure 7. One of the MOONS cameras during final alignment tests.

\subsection{Detectors}

Each of the six cameras in MOONS will use a $4 \mathrm{~K}$ x $4 \mathrm{~K}$ array that is mounted behind the field corrector lens. The four infra-red channels will exploit the new Hawiaii 4RGs from Teledyne. MOONS will use the $2.5 \mu \mathrm{m}$ cut-off, which is technically longer than is required but this maximises the QE over the MOONS wavelength range, although it does require the optical bench to be cooled to a lower temperature. The new H4RG cryogenic preamplifier has now been tested with the Engineering Grade H4RG at operational temperatures. This board allows for 64 video channels, as well as buffered outputs on the detector which helps to minimise coupling between channels and also allows for faster frame rates. Present testing indicates that the detectors can read a frame in less than 1.5 seconds, four times faster than the previous board. It is now being fully characterised and early indications suggests that the QE, dark current, read noise and the other key detector parameters are as expected, as described in [6].

In the two RI channels MOONS will use deep-depleted detectors that have been developed by Lawrence Berkeley National Laboratories (LBNL). These devices give considerably higher QE beyond $0.9 \mu \mathrm{m}$ than any other currently available $4 \mathrm{~K}$ x $4 \mathrm{~K}$ device. The LBNL Engineering Grade CCD has now achieved first light at an operational temperature of $140 \mathrm{~K}$ and it is undergoing detailed characterization.

\subsection{Observing Preparation and Path Analysis Software}

For complex instruments, and particularly for multi-object spectrometers, the usual ESO interface software is complemented by the addition of instrument-specific detailed configuration software. In MOONS, an observing preparation software (called MOONLIGHT), performs automatic allocation of fibres to science targets and sky positions, including optimisation of fibre allocations and accounting for mechanical constraints of the positioner, target priorities, and the selected observing strategy.

In order to have a high allocation efficiency of the fibres on targets, some overlap between neighbouring patrol fields is needed, with one fibre being able to patrol up to the centre of the neighbouring cell, increases the chances of collisions during positioning. To avoid such collisions, a dedicated Path Analysis Software generates collision-free trajectories for 
the fibre positioners during deployment. It receives a set of desired configurations either in advance from the Observation Preparation Software or at the telescope from the MOONS Instrument Control Software, identifies the set of positioners which can be configured successfully and returns positioner trajectories, which are sent to the fibre positioner control software. It also provides support for fibre positioner troubleshooting, determining collision-free trajectories from any configuration back to their datum location.

Following the completion of the path analysis code in $\mathrm{C}++$, further improvements have been made through subsequent testing in the context of the observing preparation software development. The baseline algorithm for the path analysis (DNF) is now ready for real FPU testing which will define the optimal FPU acceleration limits that balance the maximisation of allocation efficiency (number of FPUs able to reach their targets without conflicts) and the minimisation of collision risks for the FPUs.

\subsection{Transmission}

Since the very beginning of the project the focus (and technical challenge) has been to maximise the quality and total throughput of the instrument. The best estimates currently available for the different channels are shown in Figure 8. Note that most of the data used to generate this figure is from real data provided by suppliers for various as built components, therefore it is close to the final throughput. However, a few aspects remain to be fully quantified (on sky alignment, fibre connector losses, etc).

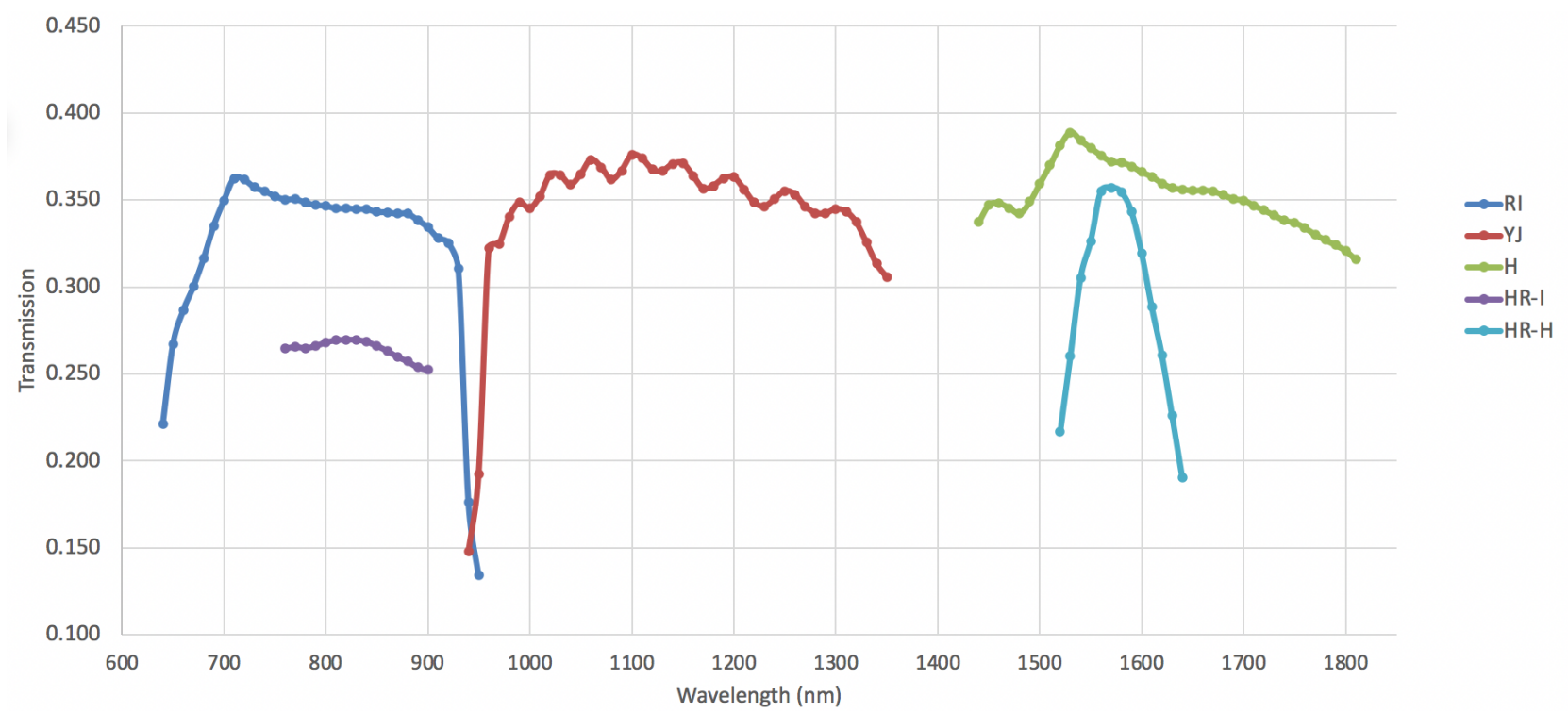

Figure 8 . The currently predicted transmission for the different MOONS channels, both low and high resolution.

\section{CONCLUSION}

The MOONS project is now in the final phase of the assembly and verification. All the major sub-systems in the frontend and the spectrograph have been manufactured and the serial production of the 1000 fibres and the fibres positioners is well underway. The expectation is to complete the project by the end of 2022 and start science operations.

\section{THANKS}

Mauricio Flores was supported by Fondo GEMINI 32130014. The Portuguese team was supported by Fundação para a Ciência e a Tecnologia (FCT, Portugal) through the research grants UID/FIS/04434/2019, UIDB/04434/2020 and UIDP/04434/2020. The Fully depleted CCDs used in MOONS were developed at the Lawrence Berkeley National 
Laboratory. The CCD development work was supported in part by the Director, Office of Science, of the U.S. Department of Energy under contract No. DE-AC02-05CH11231.

\section{REFERENCES}

[1] Cabral A.; et. Al. "MOONS, the next ESO VLT's multi-object spectrograph: The field corrector and the rotating front end"; SPIE 11447-184 (2020)

[2] Watson S., et al 'Design and testing of the MOONS fibre positioning module'; SPIE 11451-83 (2020)

[3] Black M., et al. "DMD based flat field calibration system for MOONS"; SPIE 1447-185 (2020)

[4] Guinouard I., et al. "Assemblies of the microlenses on the front-end fibres of MOONS"; SPIE 11451-247 (2020).

[5] Guinouard I., et al. "Final characteristics and performances of the fibres of MOONS"; SPIE 11451-248 (2020)

[6] Ives D. et al., "Characterisation, performance, and operational aspects of the H4RG-15 near infrared detectors for the MOONS instrument”; SPIE 11454-67 (2020) 